\title{
SUPPRESSION OF FLOW-INDUCED VIBRATIONS USING RIBBON FAIRINGS
}

\author{
JOHN M. NIEDZWECKI \& SAM M. FANG \\ Zachry Department of Civil Engineering, Texas A\&M University, College Station, Texas, USA.
}

\begin{abstract}
An experimental study was conducted to investigate the ability of ribbon fairings to suppress flow-induced vibrations on a long flexible horizontal cylinder. The test matrix included towing the cylinder at various speeds, towing the cylinder in regular waves, and investigating the influence of partial coverage on the response behavior. The test cylinder was $29 \mathrm{~m}$ long with a length to diameter (L/D) ratio of $\sim 760$. Interior to the tensioned cylinder model were six sets of unequally spaced biaxial accelerometers in a lightly pressurized environment keeping the interior dry. A string potentiometer was externally attached at the center of the model to provide a reference for later displacement estimates based on integration of the acceleration data. The time domain decomposition method (TDD) was used to recover mode shapes, damping characteristics, and modal contribution factors. For the uniform current cases, the findings illustrate that ribbon fairings are effective and provide increased damping when compared with bare cylinders. Partial coverage demonstrates that localized suppression becomes increasingly less effective as the percentage coverage is reduced. The introduction of regular waves to the towed cylinder cases illustrates the ineffectiveness of ribbon fairing to suppress the orbital motions induced by the waves, which is preferable to the amplification typically observed for airfoil fairings.

Keywords: Combined current and regular waves, horizontal flexible towed cylinder, ribbon fairings, time domain decomposition, uniform currents, vortex-induced vibrations.
\end{abstract}

\section{INTRODUCTION}

The attempt to effectively suppress vortex-induced vibrations (VIV) has lead to the invention and testing of a great many concepts and devices for offshore applications; see, for example, Zdravkovich [1], Every, King and Weaver [2] or Kumar et al. [3]. It is well known that flow-induced vibrations can be diminished by modifying the structural parameters in order to shift the natural frequency away from the range of external excitation or by disrupting organized wake formation. The performance of devices designed to suppress VIV can be quite varied depending on the structural and the environmental excitation. For example, a structure fully fitted with helical strakes can result in a 70 to $90 \%$ reduction, while when similarly fitted with airfoil-shaped fairings can result in $80 \%$ or more and reduce the drag force up to $50 \%$ based on experimental studies reported by Blevins [4]. The use of strakes and airfoil fairings can effectively reduce the vibration, although Allen et al. [5] report that strakes can increase the drag coefficient on the order of 1.5-2 or more. Additionally, Slocum et al. [6] point out that airfoil fairings may experience flutter instability issues. Every and King [7] report that a bare cylinder wrapped with circular wire can suppress $50 \%$ of the vibration at supercritical and subcritical Reynolds numbers. Owen and Bearman [8] tested a cylinder with hemispherical bumps and the vibration and drag coefficient were reduced by 47 and $25 \%$, respectively, for Reynolds numbers in the range of $10^{3}$ to $10^{4}$.

Experimental studies involving horizontal cylinders modeled at different scales have been pursued in a great many research studies investigating the performance of a wide variety of VIV suppression devices. For example, Brown and King [9] and Ding et al. [10] investigated the behavior of short cylinders fitted with suppression devices subject to both forced and free vibrations in order to evaluate lift and/or drag force coefficients and to compare their 
performance with bare cylinders. Experimental research studies investigating the flow-induced response behavior of longer horizontal cylinders are especially of interest for offshore applications, where higher mode participation, coverage density and suppression effectiveness are of particular practical interest. Frank et al. [11] tested a cylinder model with a length to diameter (L/D) ratio of 482, subject to varying uniform flow velocities. For some of the tests, the model was fitted with helical strakes whose height was $0.25 \mathrm{D}$ height and design pitch was $16 \mathrm{D}$. In those model tests, the strake coverage was varied from 0 to $100 \%$ in increments of $25 \%$. It was observed that with full coverage the root-mean-square (RMS) displacement was reduced to $0.03 \mathrm{D}$ as compared with the bare cylinder case that experienced an RMS displacement of 0.7 D. With only $75 \%$ strake coverage, the effectiveness of strakes to suppress VIV was somewhat weakened resulting in RMS displacement of $0.17 \mathrm{D}$. Vandiver et al. [12] conducted a field test with a 147.3 m length cylinder whose corresponding L/D ratio was $\sim 4145$. For $70 \%$ staggered strake coverage, it was estimated that the damage rate due to VIV was reduced by 2 orders of magnitude, while for the case of $100 \%$ coverage of strakes it was estimated that the fatigue damage was reduced by 8 orders of magnitude. Kwon et al. [13] conducted a parametric study with an approximate L/D ratio of 12 that was fitted with three fringe ribbons oriented $120^{\circ}$ apart. Their experiments showed that with a length of fringe length of $2 \mathrm{D}$, the drag coefficient was substantially reduced from about $1 \sim 1.25$ to $0.4 \sim 0.7$ in the subcritical flow regime. Nakamura and Koterayama [14] compared five different fairings in reducing the drag of towed cables whose $\mathrm{L} / \mathrm{D}$ ratio was $\sim 59$. Their findings demonstrated that ribbon fairings with a length of 7.5 D could reduce drag coefficient from 1.8 2 to 0.8.

In this study a $29 \mathrm{~m}$ long pre-tensioned horizontal cylinder with an approximate L/D ratio of 760 was used to investigate the effectiveness of ribbon fairing $(3.3 \mathrm{D})$ in reducing the multimodal vibration behavior. The instrumented model was towed at a variety of constant speeds to simulate uniform current conditions, and for some towing speeds regular waves were simultaneously run. All of the model tests were conducted in the subcritical flow regime. Zip-on ribbon fairings were introduced during some of the tests and their coverage was varied from 0 to $100 \%$ in $20 \%$ increments. Mode shapes and damping ratios for each of the dominant modes were evaluated using the time domain decomposition (TDD) technique developed by Kim et al. [15].

\section{PARTICULARS OF THE TOWED CYLINDER EXPERIMENTS}

An industrial scale experimental investigation into the flow-induced response behavior of a horizontally towed cylinder with varied fairing coverage was performed and some of that data was selected for the study reported here. The data of interest focuses on the flow-induced response behavior for model tests involving variable coverage of zip-on ribbon fairings. Additional details regarding the original scope test program can be found in the report by Chitwood [16]. Figure 1 presents a fairly detailed schematic of the test setup. The vertical I-beams shown in the figure were rigidly connected to the bridge structure and provided a means to pre-tension the cylinder model in order to target various modes of vibration. Load cells were incorporated in the design and were used to monitor the tension variations during towing. The figure also shows the location of the three wave gages and the six biaxial accelerometers that were located inside the cylinder model. The cylinder ends were sealed and a slight pressure was maintained inside the cylinder in order to keep the biaxial accelerometers dry. The cylinder support structure was designed so that the cylinder could be pre-tensioned so that the third mode would be the dominant mode of cylinder vibration. The composite cylinder had a length of $29.5 \mathrm{~m}$, an outside diameter of $38 \mathrm{~mm}$, an inside diameter of $22 \mathrm{~mm}$, 
a unit weight of $10.18 \mathrm{~N} / \mathrm{m}(0.698 \# / \mathrm{ft})$ and a mass ratio of $\sim 0.91$. Given these dimensions, the length to outer diameter, $\mathrm{L} / \mathrm{D}$, was estimated to be $\sim 760$. The cylinder depth of submergence was $0.61 \mathrm{~m}$.

The Reynolds number for the model tests was estimated to vary between $7 \times 10^{3}$ and $2.3 \times 10^{4}$ confirming that the experiments were in the subcritical flow regime. As shown in

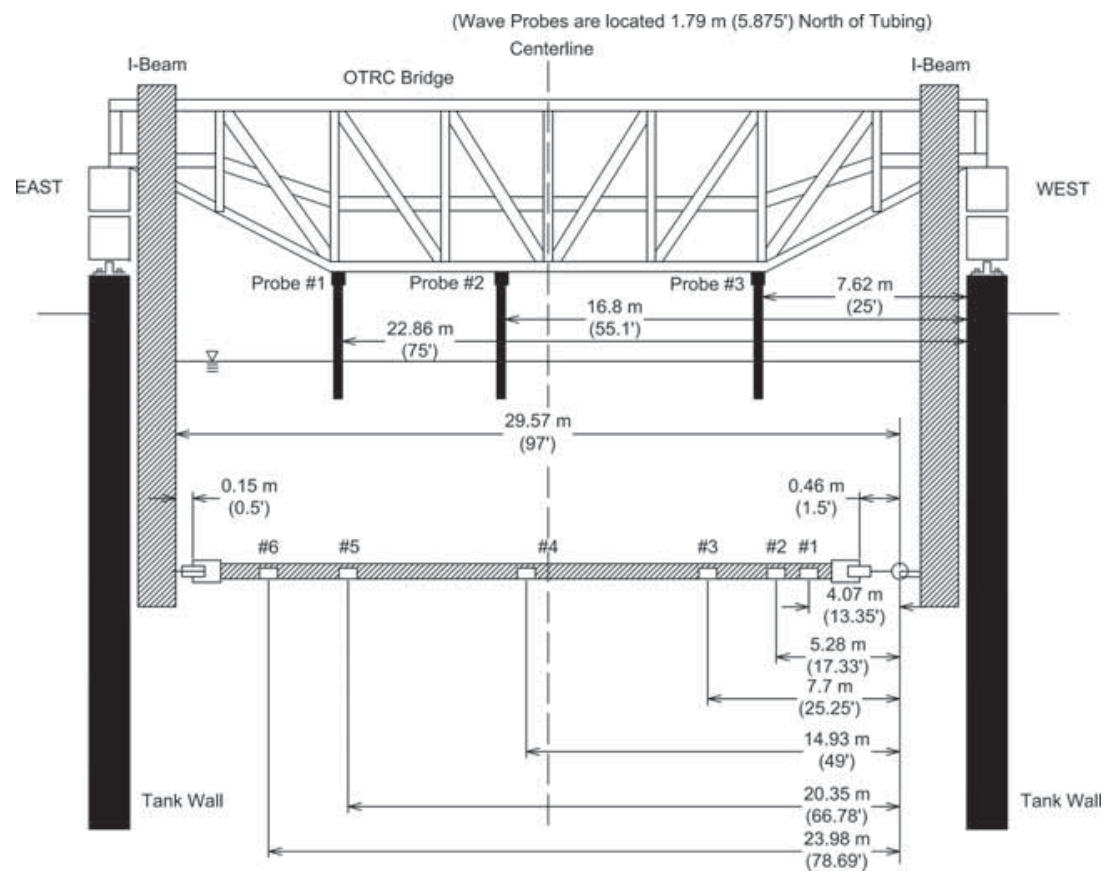

Figure 1: Schematic of the model and instrumentation locations [16].
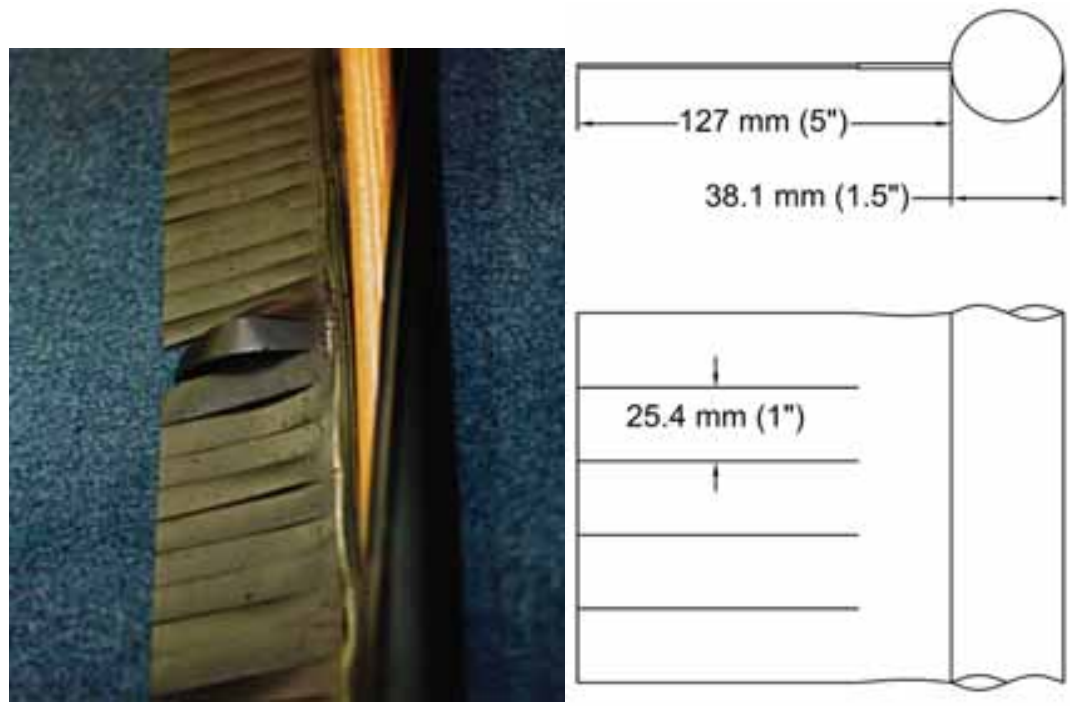

Figure 2: Ribbon fairings used in the laboratory experiments. 
Fig. 1, the six biaxial acceleration pairs are unequally spaced. A string-potentiometer sensor, not shown, was mounted on the bridge and connected to the cylinder at its mid-span in order to record the displacement. Those measurements were later used to provide a comparison with the displacement estimates obtained by the double integration of the accelerometer data. Both transverse and inline displacements were later estimated from the biaxial acceleration data.

The ribbon fairings, shown in Fig. 2, were made of a soft compliant vinyl that had an easily fastened zipper interface that was located at the front of the cylinder. The faring length when attached was 3.3 D long with a slit width of $0.67 \mathrm{D}$ at right angles to the cylinder. The fairing coverage was asymmetrically varied between 100 and $40 \%$ in $20 \%$ increments from the lefthand end of Fig. 1. The towing carriage provided uniform current velocities ranging from 0.18 to $0.43 \mathrm{~m} / \mathrm{s}$. The regular wave condition selected for this study had a wave height of 0.24 $\mathrm{m}$ and a period of $3.2 \mathrm{~s}$.

\section{TIME DOMAIN DECOMPOSITION METHOD}

A schematic diagram illustrating the relationship between the experimental measurements and the major aspects of the time domain decomposition (TDD) methodology as applied to the analysis of flow-induced vibration is presented in Fig. 3. The experimental information for the towed cylinder follows from the previous section.

The TDD method was derived and applied to data obtained from equally spaced sensors by Kim et al. [15]. In this study, the TDD method is used to analyze data from unequally spaced biaxial accelerometer measurements in order to extract mode shape, modal contributions and mode damping information. The relationship between the experiment and the TDD analysis procedure is illustrated in Fig. 3. Starting with the time history data obtained from a test run, the first step in the analysis procedure is to isolate single-degree-of-freedom signals (sdof) $[Y(i, t)]$ at specific through the application of a Butterworth filter. These vectors are then used to form an energy matrix from which one extracts the $i$ th mode shapes $\varphi_{i}$ using singular value decomposition. With this information one can also recover the damping ratio $\zeta_{i}$ and the modal contribution factor $c(i, t)$. Note that the critical damping ratios reflect the summation of structural damping and hydrodynamic damping, as in multimodal responses of a long flexible

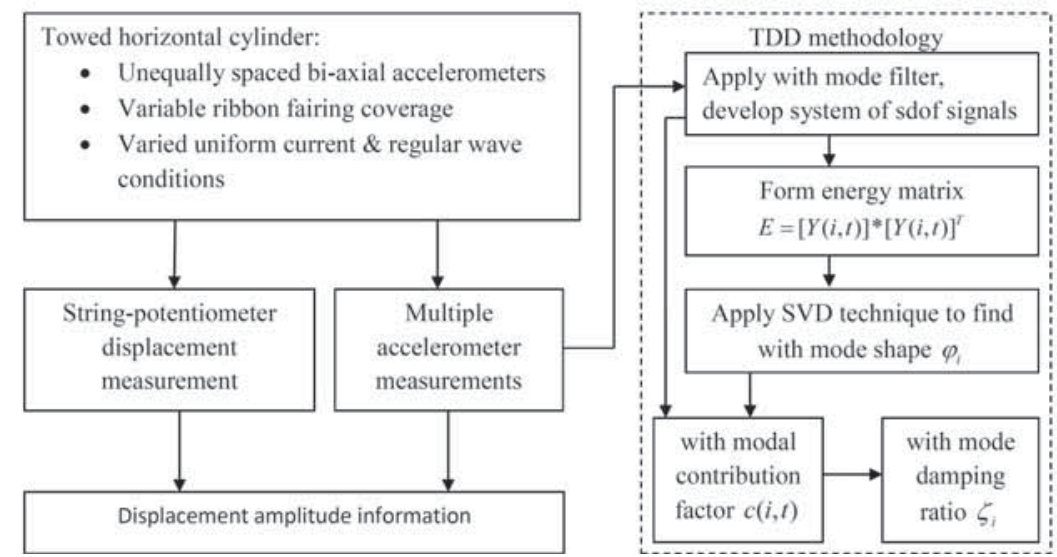

Figure 3: Schematic illustrating the connection between the model tests and TDD analysis method. 
cylinder, hydrodynamic damping could be several times larger than structural damping and cannot be neglected; see, for example, Vandiver [17].

Three features of the TDD method make it especially useful in the study of VIV problems. First the method utilizes ambient excitation for the modal analysis, unlike other methods that require free decay response (Ibrahim and Mikulcik [18]). This is essentially suitable for analyzing VIV where the excitation is not fully known and may involve, for example, combined ocean wave and sheared current flows. Second, as the size of the energy matrix is directly proportional to the number of sensors, which is why the TDD technique is an computational advantage when compared with other methods; see, for example, the method developed by Juang [19]. Finally, the TDD technique requires no assumptions regarding modal information as it depends totally on the recorded data to identify modal characteristics.

\section{ANALYSIS OF THE EXPERIMENTAL DATA}

\subsection{Horizontal cylinder response behavior with complete ribbon fairing coverage}

For the initial phase of the study the response of the towed cylinder was investigated with and without complete coverage by the ribbon fairing. The Reynolds number range for these tests was estimated to vary between $7 \times 10^{3}$ and $1.6 \times 10^{4}$, and corresponds to current velocities of 0.18 and $0.43 \mathrm{~m} / \mathrm{s}$. For the cylinder without fairings tested at constant towing speeds corresponding to current velocities $0.18,0.24,0.38$, and $0.43 \mathrm{~m} / \mathrm{s}$, the RMS amplitude ratios were determined to be $0.12,0.19,0.23$, and $0.71 \mathrm{D}$, respectively. With the introduction of the $100 \%$ coverage of the ribbon fairings along the cylinder length, those ratios were reduced to 0.05 , $0.06,0.07$, and $0.13 \mathrm{D}$. These results indicate that for the bare cylinder the RMS response increases as the uniform flow velocity increases and that the ribbon fairings are effective in significantly reducing the RMS vibration for the current velocities tested. One must be careful in characterizing the overall response behavior of the flow-induced response of a flexible cylinder with a single RMS value.

The normalized minimum and maximum flow-induced response behavior of the model in uniform current velocities of 0.24 and $0.38 \mathrm{~m} / \mathrm{s}$ are presented in Figs 4 and 5 . These figures illustrate that as the velocity is increased the dominant mode can change. Specifically, in Fig. 4 the second mode dominates the response behavior, while in Fig. 5 the third mode dominates the response behavior. In both cases it is evident that there is the influence of a higher mode contribution to each of the response envelopes near stations \#1 and \#2. At biaxial station \#5 in Fig. 5 the signal was found to be erratic, so the response behavior in that region is approximate but appears to be drawn in a manner consistent with the response observed in Fig. 4. The third mode response in Fig. 5 was confirmed using the TDD method.

Information on the critical damping ratio, dominant frequency and mode of cylinder response for three uniform current velocities are presented in Table 1. The first grouping of three columns contains estimates obtained from spectral analyses and the second groupings of three columns contain information extracted using the TDD method. In the first grouping the damping ratios were computed using the half-power bandwidth method and the frequency and mode were estimated through comparison with spectral information; see, for example, Bendat and Piersol [20]. More specifically, the most dominant mode and the corresponding frequency were estimated by selecting the largest spectral peak in the corresponding power spectral density. For the bare cylinder cases, the excited modes were obtained by comparing the spectral peak of the spectral density function. For the bare cylinder cases, the excited modes were obtained by 


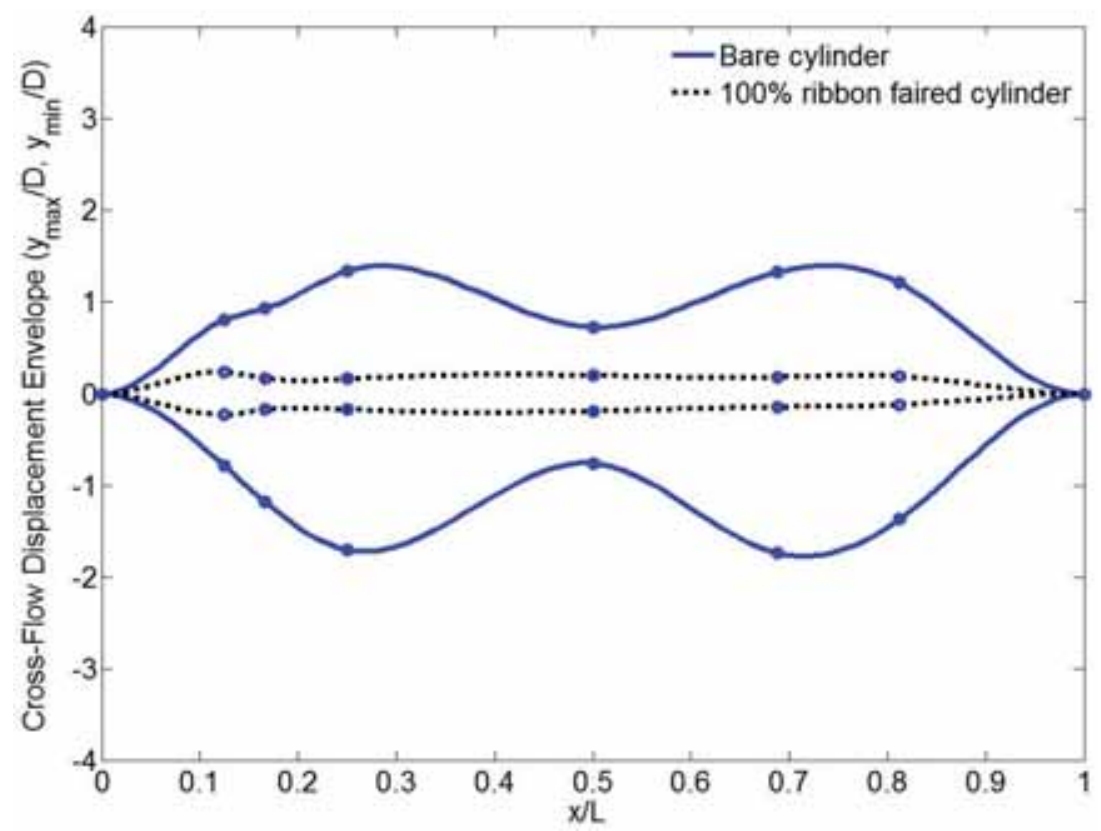

Figure 4: Cylinder response in a uniform current of $0.24 \mathrm{~m} / \mathrm{s}$.

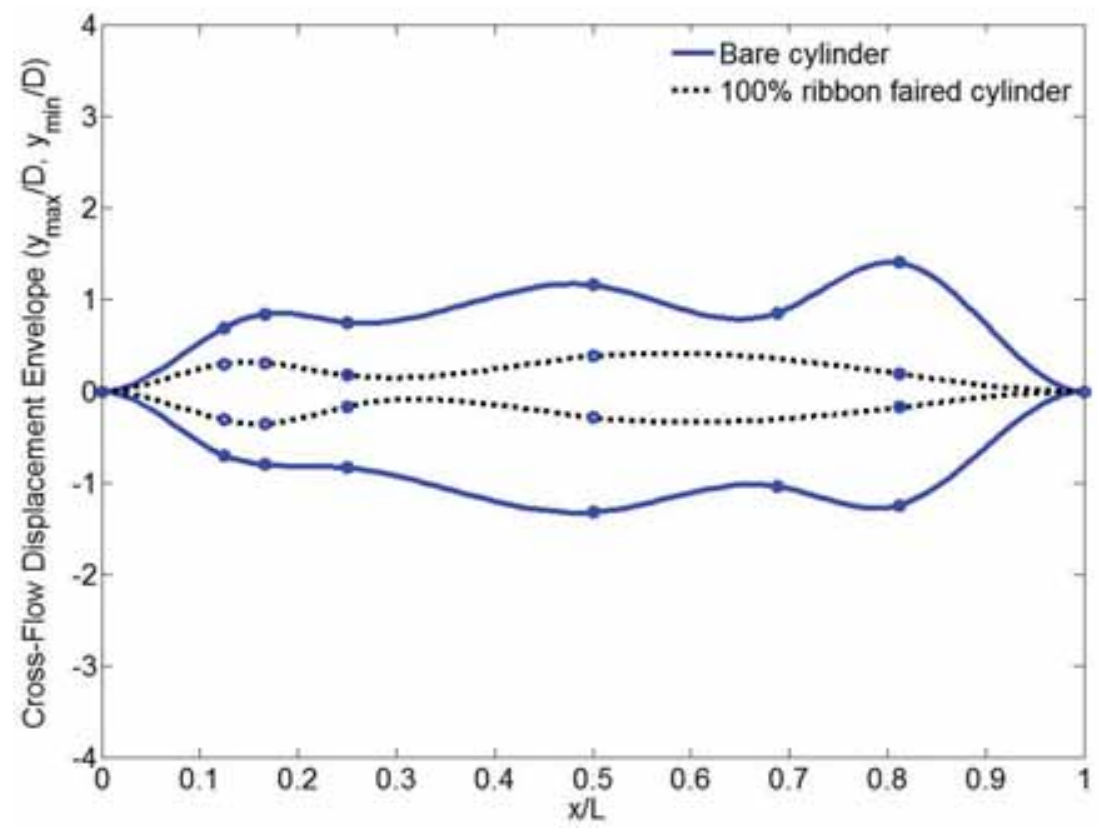

Figure 5: Cylinder response in a uniform current of $0.38 \mathrm{~m} / \mathrm{s}$. 
Table 1: Modal information obtained using spectral analysis and the TDD method.

\begin{tabular}{lccccccc}
\hline & \multicolumn{4}{c}{ Bare cylinder } & & \multicolumn{3}{c}{ Ribbon faired cylinder } \\
\cline { 2 - 3 } $\begin{array}{l}\text { Current } \\
\text { velocity }\end{array}$ & $\zeta_{i}$ & $\begin{array}{c}\text { Excited } \\
\text { frequency }\end{array}$ & $\begin{array}{c}\text { Excited } \\
\text { mode }\end{array}$ & & $\zeta_{i}$ & $\begin{array}{c}\text { Excited } \\
\text { frequency }\end{array}$ & $\begin{array}{c}\text { Excited } \\
\text { mode }\end{array}$ \\
\hline $\mathrm{m} / \mathrm{s}$ & $\%$ & $\mathrm{~Hz}$ & & & $\%$ & $\mathrm{~Hz}$ & \\
0.27 & 0.4 & 3.4 & 6 or 7 & & 4.1 & 0.8 & 2 \\
0.38 & 0.3 & 4.7 & 8 or 9 & & 2.5 & 1.3 & 3 \\
0.43 & 0.8 & 5.4 & 10 & & 1.3 & 1.4 & 3 \\
\hline
\end{tabular}

comparing the spectral peak frequencies with the natural frequency. The natural frequency was estimated based upon the known mass per unit length and measured tension and the assumption that large L/D ratio that the bending stiffness was negligible compared with the tension.

The 6 th to the 10th natural frequencies for bare cylinder were 3.29, 3.84, 4.39, 4.94, $5.48 \mathrm{~Hz}$. Thus, a mode excited at $4.7 \mathrm{~Hz}$ would indicate that the 8 th or 9 th mode would be dominant. In the TDD estimates it was not possible to identify all the modes present as the number of sensors used in the experiments limited the resolution of higher modes that could be determined from the experimental data. For the cylinder outfitted with zip-on ribbon fairing, the excited modes were confirmed by reconstructing the time series. For the cylinder outfitted with zip-on ribbon fairing, the excited modes were confirmed by reconstructing the time series using the mode shapes and modal contribution information extracted by TDD method. The dominance of the modes is validated by comparing the modal contribution factors for $c(i, t)$ each mode excited with the help of TDD method. For example, in the case of $0.43 \mathrm{~m} / \mathrm{s}$, the RMS value of $c(i, t)$ for the most dominant mode at $1.4 \mathrm{~Hz}$ is six times bigger than that of the less dominant mode at $2.8 \mathrm{~Hz}$. The modal responses listed show that for bare cylinders, the modes with the largest energy are typically excited at frequencies associated with higher modes, and for ribbon faired suppression the modes and frequencies are significantly shifted to lower frequencies modes. Damping ratios related with or without ribbon fairings is increased from $0.3-0.8$ to $1.3-4.1 \%$, respectively.

\subsection{Horizontal cylinder response to varied ribbon fairing coverage}

One the most interesting design aspects is the effect of partial coverage by the ribbon fairing on the flow-induced response. Figures 6 and 7 present a comparison of the 0,40,60, 80, and $100 \%$ ribbon fairing coverage that provide a dramatic evolutionary contrast in flow-induced response behavior. The ribbon fairing coverage was removed starting from the cylinder end near station \# 6 and was not a uniformly distributed reduction in suppression coverage. The tests were conducted with the same current velocity of $0.38 \mathrm{~m} / \mathrm{s}$. The RMS response amplitudes in the middle of cylinders at $0,40,60,80$, and $100 \%$ ribbon coverage were $0.23,0.50$, $0.36,0.26$, and $0.07 \mathrm{D}$, respectively. These values suggested that something dramatic was happening at the 40 and $60 \%$ coverage conditions and the cross-flow displacement envelopes shown in the figures further confirmed this. The 100 and the $80 \%$ coverage by the ribbon fairing were effective in reducing the amplitude of the response behavior, when compared with the bare cylinder case. 


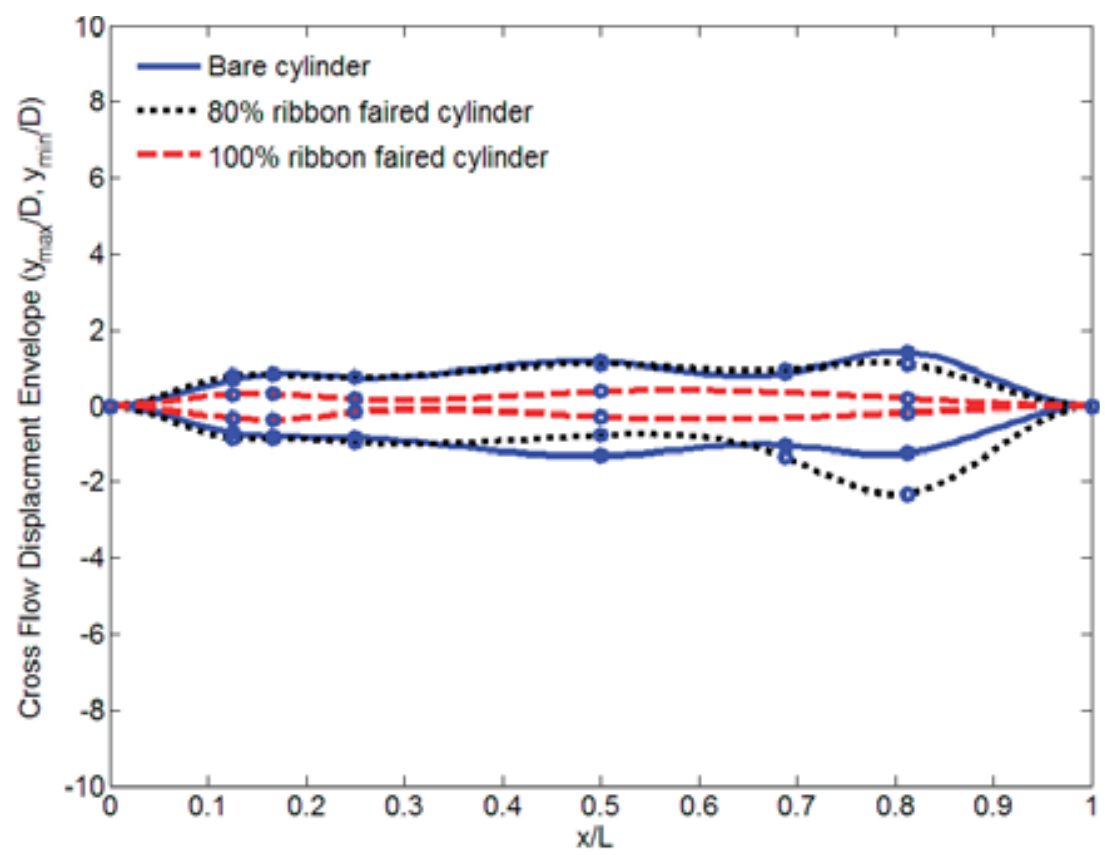

Figure 6: Cylinder response behavior with 80 and 100\% ribbon fairing coverage.

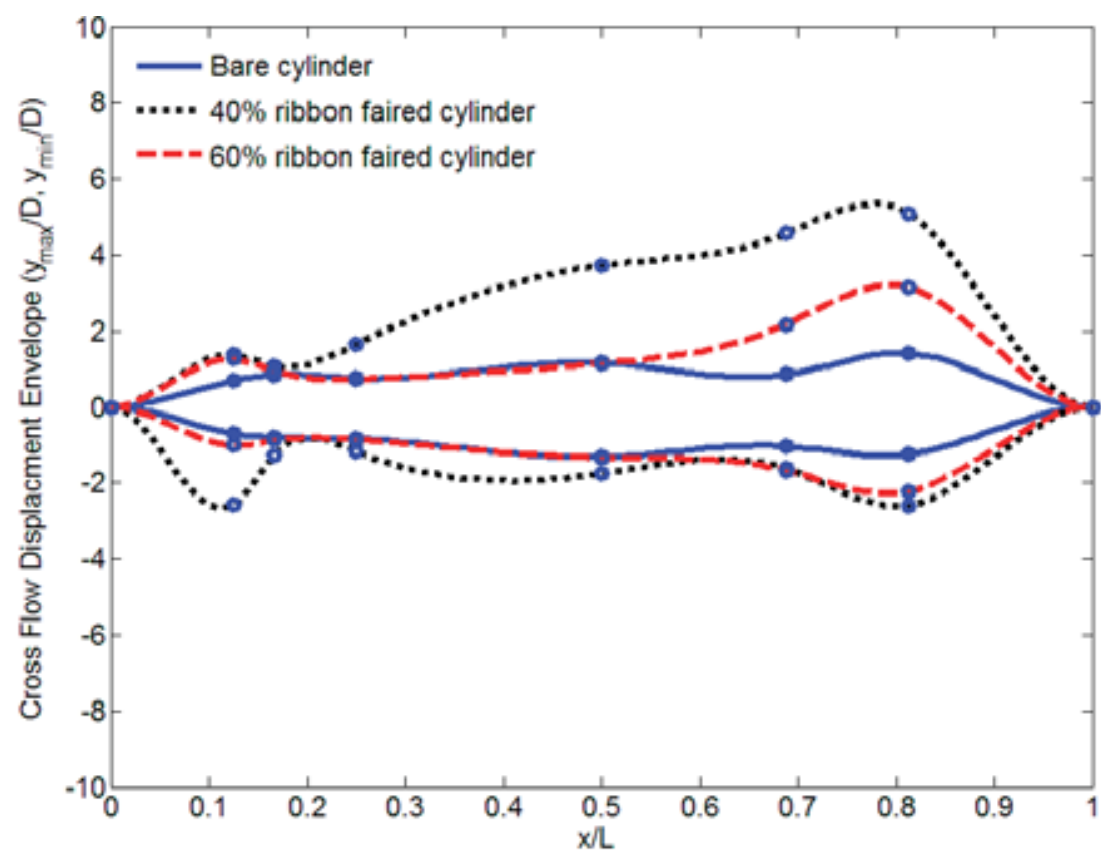

Figure 7: Cylinder response behavior with 40 and $60 \%$ ribbon fairing coverage. 


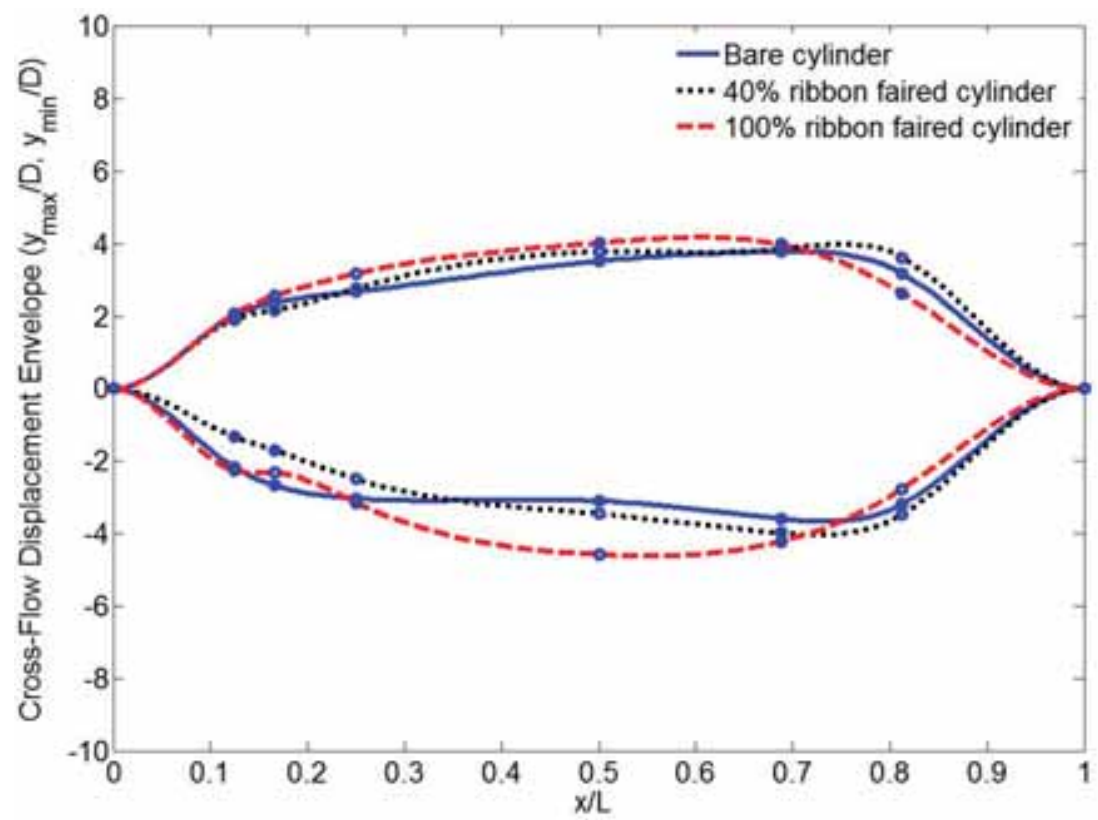

Figure 8: Horizontal cylinder response behavior in combined wave and current conditions.

However, in the $80 \%$ coverage case, there begins to be an indication of the trends seen in the 60 and $40 \%$ ribbon coverage cases. Examination of the spectral density functions is consistent with these figures indicating that energy at the higher frequencies is indeed increased and that the multimode excitation begins to dominate the response behavior.

\subsection{Combined wave and current conditions}

The flow-induced response of 0,40 , and $100 \%$ ribbon fairing coverage when combined with current and regular wave excitation are present is illustrated in Fig. 8. For comparison, the uniform current velocity was $0.38 \mathrm{~m} / \mathrm{s}$ and the wave height for the regular wave was specified as $0.24 \mathrm{~m}$. Clearly the response was dominated by the wave excitation and the RMS response amplitudes were found to be $1.86,1.80,2.08$, and $2.15 \mathrm{D}$ at $0,40,80$, and $100 \%$ coverage conditions, respectively. The fundamental mode dominates the response behavior and the presence of ribbon fairings had very little effect on the response amplitudes. However, examination of the recorded tensions at the ends of the cylinder did detect their effect. The mean values of tension being stable at $2300 \mathrm{~N}$ (520 pounds), the standard deviations of tension in coverage of $0,40,80$, and $100 \%$ were $73,68,60$ and $57 \mathrm{~N}$, respectively. The reduction of tension variation along with the increased ribbon coverage suggests that ribbon could reduce the drag force in both cross-flow and inline directions. The results are also in accordance with the conclusions of Kwon [13] and Nakamura [14], who demonstrated the drag reduction effect of ribbon fairings with experiments.

\section{SUMMARY AND CONCLUSIONS}

The TDD method in combination with spectral analysis techniques was used to interpret the flow-induced response behavior of a towed flexible horizontal cylinder with variable coverage of zip-on ribbon fairings. In the experimental study, partial coverage refers to the entire 
removal of fairings from one end of the test cylinder. So, for example, $80 \%$ coverage means that $20 \%$ of the ribbon fairings was removed from one end leaving that portion of the cylinder bare. The fairings were constructed of very lightweight vinyl and consequently added very little mass to the instrumented cylinder. For cases where the fairing coverage was either 100 or $80 \%$ and subject to uniform current flows, the ribbon fairings were shown to be very effective in suppressing VIV. As the fairing coverage was further reduced to 60 and then $40 \%$, the dynamic response was significantly changed becoming increasingly asymmetric about the uncovered portion of the test cylinder. For cylinders with large L/D ratios, flexible cylinders not dominated by bending stiffness, this finding raises some interesting questions about the dynamics of partial suppression coverage for some offshore applications. The introduction of regular waves to the towed cylinder cases illustrates that the lack of stiffness ribbon fairing material has both a negative and positive aspects for practical applications. On the positive side, the minimal relative mass and negligible rigidity of the ribbon fairings clearly does not amplify the motions as might be expected in passive airfoil suppression devices. The lack of motion suppression was countered to some degree by the observed reduction in tension variation which bears further investigation. Finally, the TDD method was shown to be effective in recovering mode shapes, damping characteristics, and modal contribution factors for data obtained from unequally spaced sensors. The relationship between the number of sensors and the ability to resolve higher modal contributions using the TDD method suggests that some optimization within practical constraints is possible.

\section{REFERENCES}

[1] Zdravkovich. M.M., Review and classification of various aerodynamic and hydrodynamic means for suppressing vortex shedding. Journal of Wind Engineering and Industrial Aerodynamics, 7(2), pp. 145-189, 1981. doi: http://dx.doi.org/10.1016/0167$\underline{6105(81) 90036-2}$

[2] Every, M.J., King, R. \& Weaver, D.S., Vortex-excited vibrations of cylinders and cables and their suppression. Ocean Engineering, 9(2), pp. 135-157, 1982. doi: http://dx.doi. org/10.1016/0029-8018(82)90010-5

[3] Kumar, R.A., Sohn, C.H. \& Gowda, B.H.L., Passive control of vortex-induced vibrations: An overview. Recent Patents on Mechanical Engineering, 1, pp. 1-11, 2008. doi: http://dx.doi.org/10.2174/2212797610801010001

[4] Blevins, R.D., 1990, Flow Induced Vibration, 2nd edn., Van Nostrand Reinhold: NY.

[5] Allen, D.W., Henning, D.L. \& Lee, L., Drilling Riser Fairing Tests at Prototype Reynolds Numbers. Proc. of the 26th Int. Conference on Offshore Mechanics and Artic Engineering, San Diego, CA, OMAE Paper No. 29219, 2007.

[6] Slocum, S.T., Ding, Z.J. \& Frank, W.R., Flutter Instability in Riser Fairings, Offshore Technology Conference, Houston, TX, OTC Paper No. 16342, 2004.

[7] Every, M.J. \& King, R., Suppression Flow Induced Vibrations - An Experimental Comparison of Clamp-on Devices, RHRA Report RR 1576, 1979.

[8] Owen, J.C. \& Bearman, P.W., Passive control of VIV with drag reduction. Journal of Fluids and Structures, 15, pp. 597-605, 2001. doi: http://dx.doi.org/10.1006/jfls. 2000.0358

[9] Brown, A.J. \& King, R., Tests with a Flexible Quasifairing to Reduce Riser Drag, Suppress VIV and Limit Drilling Down-Time, Offshore Technology Conference, Houston, TX, OTC Paper No.19161, 2008. 
[10] Ding, Z.J., Balasubramanian, S., Lokken, R.T. \& Yung, T.W., Lifting and Damping Characteristics of Bare and Straked Cylinders at Riser Scale, Offshore Technology Conference, Houston, TX, OTC Paper No. 16341, 2004.

[11] Frank, W.R., Tognarelli, M.A., Slocum, S.T., Campbell, R.B. \& Balasubramanian, S., "Flow-Induced Vibration of a Long, Flexible, Straked Cylinder in Uniform and Linearly Sheared Currents," Offshore Technology Conference, Houston, TX, OTC Paper No.16340, 2004.

[12] Vandiver, J.K., Swithenbank, S. \& Jaiswal, V., The Effectiveness of Helical Strakes in the Suppression of High Mode Number VIV, Offshore Technology Conference, Houston, TX, OTC Paper No. 18276, 2006.

[13] Kwon S.H., Cho, J.W., Park, J.S. \& Choi, H.S., The effects of drag reduction by ribbons attached to cylindrical pipes. Ocean Engineering, 29, pp. 1945-1958, 2002. doi: http:// dx.doi.org/10.1016/S0029-8018(02)00010-0

[14] Nakamura, M. \& Koterayama, W., A study on cable fairing. Proc. of the 2nd Int. Offshore \& Polar Engineering Conference, San Francisco, CA, pp. 301-307, 1992.

[15] Kim, B.H., Stubbs, N. \& Park, T., A new method to extract modal parameters using output-only responses. Journal of Sound and Vibration, 282, pp. 215-230, 2005. doi: http://dx.doi.org/10.1016/j.jsv.2004.02.026

[16] Chitwood, J.S., Vortex-induced vibration of a slender horizontal cylinder in currents and waves, OTRC report No. 2/98-A9575, 1998.

[17] Vandiver, J.K., Dimensionless parameters important to the prediction of vortex-induced vibration of long, flexible cylinders in ocean currents. Journal of Fluids and Structures, 7 , pp. 423-455, 2003. doi: http://dx.doi.org/10.1006/jfls.1993.1028

[18] Ibrahim, S.R. \& Mikulcik, E.C., A Method for the direct identification of vibration parameters from the free response. Shock and Vibration Bulletin, 47(4), pp. 183-198, 1977.

[19] Juang, J.N., Applied System Identification, Prentice-Hall, Englewood Cliffs: New York, 1994.

[20] Bendat, J.S. \& Piersol, A.G., Engineering Applications of Correlation and Spectral Analysis, Wiley: New York, 1980. 